\title{
Consumo de heroína en Colombia, prácticas relacionadas e incidencia en la salud pública
}

\author{
Heroin consumption in Colombia-associated practice and \\ incidence in the public health care
}

\author{
Guillermo Alonso Castaño Pérez ${ }^{\text {I }}$ Gustavo Adolfo Calderón Vallejo ${ }^{\text {II }}$ \\ IMédico Especialista en Farmacodependencia. Máster en Drogodependencias. \\ Fundación Universitaria Luis Amigó. Medellín, Colombia. \\ ${ }^{\text {II } S o c i o ́ l o g o . ~ M a ́ s t e r ~ e n ~ E s t u d i o s ~ U r b a n o s . ~ D o c e n t e ~ I n v e s t i g a d o r . ~ F u n d a c i o ́ n ~}$ \\ Universitaria Luis Amigó. Medellín, Colombia.
}

\section{RESUMEN}

Introducción El consumo de heroína empieza a ser una amenaza para la salud pública de Colombia, entre otras razones, porque los consumidores la están adquiriendo a más bajo costo.

Objetivos Caracterizar el consumo de heroína en la ciudad de Medellín y su área metropolitana.

Métodos Investigación cualitativa. La información se recolectó entre los meses de julio y septiembre de 2008. El acceso a los participantes se hizo a través de los centros de tratamiento del consumo de drogas y mediante la técnica de "bola de nieve". La población quedó constituida por 42 consumidores regulares de heroína a quienes se les aplicó una entrevista estructurada, cara a cara, que consultó por el tipo de heroína consumida, vías de administración, frecuencias de consumo, utensilios utilizados, prácticas de consumo, rituales que acompañan estas prácticas, relaciones sexuales y protección, ideas y gestos suicidas.

Resultados Los consumidores eran predominantemente hombres, solteros, con edades comprendidas entre los 18 y los 23 años, sin hijos y muchos con estudios universitarios no terminados. Mayoritariamente se ubicaban en un estrato socioeconómico medio, tenían antecedentes familiares de consumo de sustancias psicoactivas, lo que favoreció su inicio en el consumo de drogas en edades tempranas. La principal vía de uso de la heroína era la inhalación, aunque también la consumen inyectada y fumada. Los consumidores comparten jeringas y otros utensilios, tienen relaciones sexuales bajo el efecto de las drogas sin condón y 
delinquen para poder suministrarse la sustancia.

Conclusiones El consumo de heroína es una realidad emergente en Colombia y su área metropolitana, que acecha de manera importante a la salud pública, la seguridad y la convivencia. Es necesario hacer estudios específicos de prevalencia e incidencia de esta sustancia que permita tomar urgentes medidas de intervención.

Palabras clave: Heroína, Medellín, Colombia, salud pública.

\begin{abstract}
Introduction Heroin addiction represents a threat to the public health in Colombia because, among other reasons, the heroin users are purchasing the drug at lower prices.

Objectives To characterize the heroin consumption in Medellín and its metropolitan areas.

Methods Qualitative research study. Information collected from July to September, 2008. The participants were accessed through the drug addiction treatment centers and the "snowball" technique. The population was finally made up of 42 sustained heroin users, who were administered a face-to-face structured interview that asked about type of heroin used, routes of administration, frequency, implements, practices, accompanying rituals, sexual relations and use of protection, suicidal ideas and gestures.

Results Heroin users were predominantly single males aged 18-23 years, having no children and unfinished university studies. They belonged to middle socioeconomic stratum, had a history of use of psychoactive substances, which prompted the use of drugs at early ages. The main route of administration was intranasal, although heroin injection and smoking were also present. The drug users shared syringes and other implements; they had unprotected sexual intercourse under the effects of drug and committed crimes to afford heroin. Conclusions Heroin use is emerging reality in Colombia and its metropolitan area, and represents a significant threat to public health, safety and conviviality. It is required to conduct specific prevalence and incidence studies of this substance in order to take immediate intervention actions.
\end{abstract}

Key words: Heroin, Medellin, Colombia, public health.

\title{
INTRODUCCIÓN
}

La heroína, una de las sustancias psicoactivas más adictivas que ha conocido la historia de la humanidad, atractiva por el aparente bienestar que causa, al que sigue la instauración de un proceso de tolerancia y dependencia que afecta gravemente las esferas biológica, psicológica y social de los consumidores, empieza a ser una amenaza para la salud pública de Colombia. La presencia de cultivos de amapola, que en el país alcanzaron para el año 2008, 714 hectáreas, según informe del Observatorio de Drogas de Colombia, ${ }^{1}$ ha incidido en el aumento de la disponibilidad de la sustancia en el mercado callejero y, por lo tanto, los consumidores están teniendo fácil acceso a la droga y la adquieren a bajos costos. 
Lo anterior se refleja en que los índices de prevalencia en el consumo de esta sustancia empiezan a aumentar. Todo esto, sumado a las malas prácticas en el consumo, como el intercambio de jeringuillas y la mezcla de aditamentos diferentes, hace que la amenaza no sea un fantasma, sino una realidad.

Con respecto a las prevalencias del consumo de heroína en Colombia, existen algunos estudios, que si bien no son comparables por las diferentes muestras de población y metodologías empleadas, sí dan una idea de su aumento (tabla 1).

Tabla 1. Cuadro epidemiológico de consumo de heroína en Colombia

\begin{tabular}{|c|c|}
\hline Estudio & $\begin{array}{l}\text { Prevalencia en } \\
\text { población general }\end{array}$ \\
\hline $\begin{array}{l}\text { Segundo Estudio Epidemiológico de } \\
\text { Consumo de Sustancias Psicoactivas de } \\
\text { la ciudad de Santa Fe de Bogotá. } 1992 .^{2}\end{array}$ & $\begin{array}{l}\text { Prevalencia vida: } 2 \\
\text { por mil. } \\
\text { Prevalencia anual: } \\
0,6 \text { por mil. }\end{array}$ \\
\hline $\begin{array}{l}\text { Primer Estudio Nacional de Salud Mental } \\
\text { y Consumo de Sustancias Psicoactivas. } \\
1993 .^{3}\end{array}$ & $\begin{array}{l}\text { Prevalencia vida: } \\
0,5 \text { por mil. } \\
\text { Prevalencia anual: } \\
0,6 \text { por mil. }\end{array}$ \\
\hline $\begin{array}{l}\text { Estudio Nacional sobre Consumo de } \\
\text { Sustancias Psicoactivas. } 1996^{4}\end{array}$ & $\begin{array}{l}\text { Prevalencia vida: } \\
0,4 \text { por mil. }\end{array}$ \\
\hline $\begin{array}{l}\text { Segundo Estudio Nacional de Salud } \\
\text { Mental y Consumo de Sustancias } \\
\text { Psicoactivas. } 1997^{5}\end{array}$ & $\begin{array}{l}\text { Prevalencia anual: } \\
12 \text { por mil. }\end{array}$ \\
\hline $\begin{array}{l}\text { Sondeo Nacional sobre Consumo de } \\
\text { Drogas en Jóvenes. Programa } \\
\text { Presidencial Rumbos. Comisión Nacional } \\
\text { de Investigación sobre Drogas. } \\
\text { Presidencia de la República de Colombia. } \\
1999 .^{6}\end{array}$ & $\begin{array}{l}\text { Prevalencia anual } \\
\text { ciudad de Yopal: } 28 \\
\text { por mil (4 654 } \\
\text { encuestados). } \\
\text { Prevalencia anual } \\
\text { ciudad de Puerto } \\
\text { Carreño: } 21 \text { por mil } \\
\text { ( } 817 \text { encuestados). } \\
\text { Prevalencia anual } \\
\text { ciudad de Ibagué: } \\
11 \text { por mil (9 } 612 \\
\text { encuestados). } \\
\text { Prevalencia anual } \\
\text { ciudad } \\
\text { de Medellín: } 11 \text { por } \\
\text { mil (19 } 213 \\
\text { encuestados). }\end{array}$ \\
\hline $\begin{array}{l}\text { Sistema de Vigilancia Epidemiológica del } \\
\text { Consumo de Sustancias Psicoactivas- } \\
\text { VESPA-. Formato para Centros } \\
\text { Penitenciarios. Unidad de Prevención } \\
\text { Integral del Departamento de Caldas. } \\
\text { Gobernación de Caldas. Manizales, } \\
\text { 1999.7 }\end{array}$ & $\begin{array}{l}\text { Prevalencia vida: } 2 \\
\text { por mil. }\end{array}$ \\
\hline $\begin{array}{l}\text { VESPA UNIVERSITARIO. Sistema de } \\
\text { Vigilancia Epidemiológica sobre }\end{array}$ & $\begin{array}{l}\text { Prevalencia vida: } 34 \\
\text { por mil. }\end{array}$ \\
\hline
\end{tabular}




\begin{tabular}{|c|c|}
\hline $\begin{array}{l}\text { Consumo de Sustancias Psicoactivas en } \\
\text { Población Universitaria. Fundación } \\
\text { Universitaria Luis Amigó. Red } \\
\text { Universitaria en Farmacodependencia - } \\
\text { Red Unir-. Secretaría de Salud de } \\
\text { Medellín. Medellín, 2000. }\end{array}$ & $\begin{array}{l}\text { Prevalencia anual: } \\
31 \text { por mil. }\end{array}$ \\
\hline $\begin{array}{l}\text { Encuesta Nacional sobre Consumo de } \\
\text { Sustancias Psicoactivas en Jóvenes de } \\
10 \text { a } 24 \text { años. Colombia, } 2001 . \\
\text { Programa Rumbos. Presidencia de la } \\
\text { República. }{ }^{9} \text { Prevalencia vida: } 11 \text { por mil. }\end{array}$ & $\begin{array}{l}\text { Prevalencia anual: } 6 \\
\text { por mil. }\end{array}$ \\
\hline $\begin{array}{l}\text { Estudio Mundial sobre Uso de Drogas } \\
\text { Inyectadas y Consecuencias para la } \\
\text { Salud de la OMS, realizado en dos fases } \\
\text { entre } 2001 \text { y } 2002 \text {, Bogotá. (Mejía \& } \\
\text { Pérez, 2003). }{ }^{10} \text { (N: 382). }\end{array}$ & $\begin{array}{l}\text { Prevalencia de vida, } \\
\text { primera fase el } 71,8 \\
\%(N=51) \text { inyección } \\
\text { de heroína frente al } \\
20,3 \% \text { de la } \\
\text { segunda fase } \\
(\mathrm{N}=301) .\end{array}$ \\
\hline $\begin{array}{l}\text { Estudio de Consumo de Sustancias } \\
\text { Psicoactivas en Población Penitenciaria. } \\
2006 \text { (Evaluación del Progreso de } \\
\text { Control de Drogas. 2005-2006. } \\
\text { Colombia. MEM. CICAD/OEA). }{ }^{11}\end{array}$ & $\begin{array}{l}\text { (N: } 61356) \text {. } \\
\text { Prevalencia vida: } 25 \\
\text { por mil. } \\
\text { Prevalencia anual: } \\
19 \text { por mil. }\end{array}$ \\
\hline $\begin{array}{l}\text { Reporte de algunos centros de } \\
\text { tratamiento en Colombia. } 2005 . \\
\text { (Reportes personales). }\end{array}$ & 153 casos. \\
\hline $\begin{array}{l}\text { Reporte de algunos centros de } \\
\text { tratamiento. Primer trimestre año } 2006 . \\
\text { (Reportes personales). }\end{array}$ & $\begin{array}{l}64 \text { consultas por } \\
\text { consumo de } \\
\text { heroína. }\end{array}$ \\
\hline $\begin{array}{l}\text { Reporte de pacientes tratados en } \\
\text { Empresa Social del Estado CARISMA, en } \\
\text { la ciudad de Medellín } 2003-2008 .^{12}\end{array}$ & $\begin{array}{l}73 \text { personas } \\
\text { hospitalizadas por } \\
\text { consumo de } \\
\text { heroína, } \\
\text { correspondiendo el } \\
\text { mayor número de } \\
\text { usuarios a los } \\
\text { últimos } 3 \text { años. }\end{array}$ \\
\hline $\begin{array}{l}\text { Estudio Nacional sobre Consumo de } \\
\text { Sustancias Psicoactivas en Colombia. } \\
\text { 2008. Ministerio de la Protección Social, } \\
\text { Dirección Nacional de Estupefacientes. }{ }^{13}\end{array}$ & $\begin{array}{l}\text { (N=29164 } \\
\text { personas). } \\
\text { Prevalencia de vida: } \\
19 \text { por mil. } \\
\text { Prevalencia anual: } 2 \\
\text { por mil. } \\
\text { Prevalencia mes: } 2 \\
\text { por mil. }\end{array}$ \\
\hline
\end{tabular}

Fuente: tabla construida por los autores a partir de las fuentes enumeradas en la columna de la izquierda. 
El consumo de drogas ilícitas, como la heroína, expone a los usuarios a una variedad de problemas de salud agudos y crónicos. Las complicaciones médicas asociadas con el uso de este tipo de sustancias incluyen los siguientes problemas: a) Ios relacionados con sus propiedades farmacológicas: sobredosis, síndromes de abstinencia, lesiones por accidentes bajo su influencia, dependencia; b) los relacionados con sus vías de administración: hepatitis, abscesos e infecciones, celulitis y el VIH/sida por el uso de jeringas no esterilizadas; y c) los relacionados con sus actitudes: conductas delictivas, problemas familiares, abandono escolar y desempleo. Estos problemas traen consigo serias implicaciones para la salud tanto individual como pública, y debe tenerse en cuenta que muchos de ellos pueden ser tratados y todos son prevenibles. ${ }^{14}$

El objetivo de este artículo es realizar un análisis del uso de esta droga en un grupo de consumidores de la ciudad de Medellín y su área metropolitana, las prácticas relacionadas con ese consumo y su posible incidencia en la salud pública; análisis que permita desarrollar propuestas de promoción e intervención y anime a otros investigadores a continuar los estudios acerca del tema para adelantarse a un problema que ya ha diezmado a otras sociedades como, por ejemplo, la española, donde, según un balance provisional de la epidemia de heroína, las cifras de consumo resultan muy altas. Con los datos publicados, ${ }^{15-19}$ se estima que unas 212 000 personas han sido tratadas por dependencia de esta droga en centros que notifican al indicador tratamiento del Plan Nacional de Drogas español, por lo que los usuarios problemáticos deben haber sido más de 300 000. Unos 100000 inyectores de drogas (prácticamente todos inyectores de heroína) se han infectado por VIH y bastantes más por (virus de hepatitis). Finalmente, se han producido entre 20000 y 25000 muertes por sobredosis o reacción aguda a drogas en más del $90 \%$ de los casos con implicación de heroína. ${ }^{20}$

\section{MÉTODOS}

La presente investigación responde a un diseño cuantitativo-cualitativo, mediante el estudio de casos múltiples. El trabajo de campo se llevó a cabo en la ciudad de Medellín y en su área metropolitana, entre los meses de julio y septiembre de 2008. Es bien sabido el difícil acceso que se tiene, en general, a la población consumidora de drogas y más a usuarios de heroína, por ser aún un grupo minoritario y consumir una droga apenas emergente en Colombia. El reclutamiento de participantes para el estudio se realizó a través de los centros de tratamiento del consumo de drogas que existen en la ciudad y por medio de la técnica de "bola de nieve."

La selección fue intencional y la muestra quedó conformada por 42 personas consumidoras regulares de heroína.

Se utilizó una entrevista estructurada, cara a cara, con un tiempo de realización de 40 min. El cuestionario recogía información necesaria para dar cuenta de las características de este grupo de consumidores y de su consumo. Además de algunos datos demográficos, se solicitó información sobre la edad de inicio del consumo, patrones y frecuencia de consumo de heroína en el momento de la entrevista, tratamientos realizados y situación socio-familiar.

Las entrevistas se realizaron alternativamente por cuatro profesionales: una especialista en farmacodependencia y tres psicólogos ya graduados y en formación para esta misma especialidad, todos conocedores del tema y del universo de estudio en la ciudad de Medellín y en su área metropolitana. Las entrevistas se 
concertaron con los directores de los centros de tratamiento y las personas consumidoras de heroína que estaban internadas en ellos. Para el caso de los contactos obtenidos a través de la técnica "bola de nieve", se concertó un lugar para la entrevista. No se entregó ninguna retribución por contestarla.

Los análisis se llevaron a cabo con el paquete estadístico Atlas ti, versión 5.5, para Windows.

El mayor inconveniente de este estudio fue la dificultad para captar participantes. Aunque se hizo un gran esfuerzo (exploración de múltiples escenarios, contacto con gran número de captadores), hubo grandes dificultades para reunir la muestra que finalmente se obtuvo. En todos los casos, la información se llevó hasta saturar cada una de las variables propuestas en el estudio.

\section{RESULTADOS}

\section{Perfil de los consumidores}

En esta investigación se encontró que los consumidores de heroína son predominantemente hombres solteros, con edades comprendidas entre los 18 y los 23 años aproximadamente, sin hijos y muchos con estudios universitarios no terminados (tabla 2).

Mayoritariamente se ubican en un estrato socioeconómico medio, tienen antecedentes familiares de consumo de sustancias psicoactivas y se iniciaron en el consumo de drogas a edades tempranas, aproximadamente entre los 13 y los 15 años; todos presentan historia de policonsumo de sustancias psicoactivas, entre las que cabe incluir el alcohol, el tabaco, la marihuana, la cocaína, los alucinógenos, las benzodiacepinas y algunas drogas recreativas como el popper y el éxtasis. En cuanto a la heroína, comenzaron a usarla en su mayoría (71\%) entre los 15 y los 20 años.

También se indagó acerca de los antecedentes de consumo de sustancias psicoactivas en la familia de los consumidores entrevistados. Casi la totalidad de los participantes, $88 \%$ de la muestra, declaró tener antecedentes familiares y parentales en el consumo de drogas, incluyéndose en este grupo a abuelos, tíos, primos, padres y hermanos, quienes consumían tabaco, alcohol, marihuana, y cocaína. En la tabla 2 se pueden apreciar las características sociodemográficas y del consumo de heroína. 
Tabla 2. Descriptiva de variables saciodemográficas (N=42)

\begin{tabular}{|c|c|}
\hline Variables & Resultados \\
\hline $\begin{array}{l}\text { Edad de inicio en el consumo } \\
\text { de heroina }\end{array}$ & $\begin{array}{l}36 \text { \% (15) entre los } 15 \text { y los } 17 \text { años. } \\
35 \text { \% (16) entre los } 18 \text { y los } 20 \text { años. } \\
29 \text { \% (11) con más de } 20 \text { años. }\end{array}$ \\
\hline Edad de los consumidores & $\begin{array}{l}59.5 \% \text { (25) con edades entre los } 18 \text { y los } 23 \\
\text { afios. } \\
26.1 \% \text { (11) entre los } 24 \text { y los } 29 \text { años. } \\
14.2 \% \text { (6) entre los } 30 \text { y los } 35 \text { años. }\end{array}$ \\
\hline Sexo & $\begin{array}{l}80.9 \% \text { (34) hombres. } \\
19.1 \% \text { (8) mujeres. }\end{array}$ \\
\hline Estado civil & $\begin{array}{l}87 \%(37) \text { solteros. } \\
11 \%(4) \text { casados. } \\
2 \% \text { (1) viudo. }\end{array}$ \\
\hline Nivel educativo & $\begin{array}{l}38 \% \text { con bachillerato, (0.5 completo, } 11 \\
\text { incompleto). } \\
9.5 \% \text { (4) estudias técnicos. } \\
45 \% \text { (19) con estudias universitarios en } \\
\text { cuss. } \\
2 \%(1) \text { sin estudios. } \\
4 \%(2) \text {. No respondieron. }\end{array}$ \\
\hline Nivel socioeconómico & $\begin{array}{l}42 \% \text { (18) medio bajo. } \\
40 \%(17) \text { estrato medio. } \\
15 \% \text { (6) estrato bajo. } \\
3 \% \text { (1) estrato alto. }\end{array}$ \\
\hline $\begin{array}{l}\text { Antecedentes familiares } \\
\text { de consumo de drogas }\end{array}$ & $899(37)$ con antecedentes. \\
\hline
\end{tabular}

Fuente: los autores.

\section{Tipo y calidad de la heroína consumida}

Teniendo en cuenta lo descrito por Stockley, ${ }^{21}$ y confirmado por Gómez, ${ }^{22}$ con respecto a la forma como se clasifican las heroínas de acuerdo con su grado de pureza, podría afirmarse, por los relatos obtenidos de los consumidores de heroína entrevistados, que en Medellín y su área metropolitana se están consiguiendo los tres tipos de esta droga: marrón, blanca y negra.

\section{Vías de administración, dosis y frecuencia de consumo}

La mayoría de los pacientes entrevistados (24), usaban la vía inhalada, 10 de ellos dijeron que probaron y se quedaron con la vía intravenosa, mientras que 8 alternaban la vía inhalada y fumada. Algunos han incursionado en las tres vías, 7 de ellos han probado la heroína fumada, inhalada e inyectada; 6 dijeron que en algún momento la habían inhalado y fumado, y 5 expresaron que la habían inhalado e inyectado.

De otro lado, a la hora de expresar las razones para preferir una vía determinada para administrarse la heroína, se dieron en las respuestas dos tendencias:

- De los que no se inyectan (32), 9 prefirieron no hacerlo porque le tenían miedo a las agujas, 8 confirmaron que la vía intravenosa es más peligrosa que las demás, 4 consideraron que al inyectarse se vuelven más adictos, 4 dijeron que consumiendo 
de esta forma tenían más riesgos de una sobredosis o de morir mientras dormían y 2 opinaron que el síndrome de abstinencia es mayor. Finalmente, 5 de las personas entrevistadas manifestaron explícitamente que no se inyectaban porque hacerlo dejaba marcas en el cuerpo y que, conocían a otros consumidores que lo hacían y que ya tenían las señales de la "degradación", y esto los alejaba de esta vía.

-Los que iniciaron el consumo por vía inhalada y luego pasaron a la vía intravenosa argumentaron que lo hicieron porque ya su cuerpo había generado una tolerancia a los efectos de la heroína que los llevaba a incursionar en otras formas de administración para volver a sentir las sensaciones iniciales que les había brindado la droga. Así mismo, hay otros que dijeron que se pasaron a la vía intravenosa porque consumiendo de esta forma necesitaban una menor cantidad de dosis y, por lo tanto, ahorraban dinero.

En cuanto a las dosis consumidas, las tendencias en el análisis de los datos suministrados por los 42 entrevistados, dan cuenta de que alrededor del $21 \%$ (9) consume en promedio hasta $0,25 \mathrm{~g} /$ día; el $25 \%$ (11), entre 0,5 y $0,75 \mathrm{~g} /$ día; $34 \%$ (14), $1 \mathrm{~g} /$ día; el $8 \%$ (3), $2 \mathrm{~g} /$ día; y el $12 \%$ (5) consumían más de $2 \mathrm{~g} /$ día. La frecuencia de consumo por la vía inhalada, la más alta, era como promedio cada 2$3 \mathrm{~h}$; cuando la vía utilizada era la intravenosa, era más espaciada, cada 6-8 h comompromedio.

Datos sobre la vía, dosis y frecuencia de administracion pueden ser observados en la tabla 3.

Tabla 3. Vías de administración, dosis y frecuencia de consumo

$$
(\mathrm{N}=42)
$$

\begin{tabular}{|l|l|}
\hline Variable & \multicolumn{2}{|c|}{ Resultado } \\
\hline Vía de administración & $57 \%(24)$ vía inhalada. \\
& $24 \%(10)$ vía intravenosa. \\
& $19 \%(8)$ alternan vía inhalada y fumada. \\
\hline Cantidad & $34 \%(14) 1 \mathrm{~g} /$ día. \\
& $25 \%(11)$ entre 0,5 y $0,75 \mathrm{~g} /$ día. \\
& $21 \%(9) 0,25 \mathrm{~g} /$ día. \\
& $12 \%(5)$ consumen más de $2 \mathrm{~g} / \mathrm{día}$. \\
& $8 \%(3)$ dos g/día. \\
\hline Frecuencia de consumo & Vía inhalada-fumada: promedio cada $2-3 \mathrm{~h}$. \\
& Vía intravenosa: promedio cada $6-8 \mathrm{~h}$. \\
\hline
\end{tabular}

Fuente: los autores.

Con respecto a las facilidades para conseguir la heroína, aunque los entrevistados en general sugirieron que es una droga de acceso restringido, también despuntó entre sus respuestas la facilidad con que la consiguen. En este sentido, en 34 ocasiones los entrevistados respondieron con frases como las siguientes: "es fácil porque se conoce a las personas que la venden", "era muy fácil conseguirla", "fácil conseguirla porque es solo una llamada telefónica" y "era fácil porque tenía los contactos."

\section{Prácticas de consumo}


El total de entrevistados tuvo en algún momento prácticas colectivas en el consumo de heroína, la mayoría de los consumidores atribuyeron esta preferencia a que en el grupo se puede compartir el gasto de la compra de la dosis, mantener rituales comunes $y$, así mismo, obtener un respaldo o ayuda frente al riesgo de una sobredosis. 27 (64\%) de los pacientes entrevistados consumían con amigos, mientras $9(21 \%)$ consumían heroína con sus parejas como una forma de establecer el vínculo amoroso, cuidarse entre ellos y experimentar relaciones sexuales placenteras, en la mayoría de tales casos.

En cuanto a los rituales de consumo, el total de entrevistados tuvo en algún momento prácticas colectivas; quienes no compartieron los utensilios necesarios para la práctica, aseguraron haber observado intercambio de jeringas y elementos para el consumo. En lo relatado por los entrevistados, se presentaron dinámicas de consumo comunes, entre ellas:

-Algunos de los consumidores se inyectan entre sí, se pasan el humo de boca a boca o comparten la bolsita y el pitillo para inhalar.

-Algunos tienen prácticas sexuales en medio del consumo.

Entre los que intercambian jeringas, las razones que argumentaron para ello son múltiples: ansiedad, desespero por los síntomas del síndrome de abstinencia o simplemente lo hacen en forma exclusiva con personas que conocen previamente y con quienes "tienen confianza". Es de notar también que en algunos casos los pacientes manifestaron que, aunque a veces las jeringas no se prestaran, sí es muy común reutilizarlas. Los siguientes testimonios pueden dar idea de las percepciones de los usuarios en relación con el intercambio de aditamentos durante los consumos de esta droga:

Lo único que no se presta es la jeringa y es por higiene $y$, más que todo, por el miedo, por el miedo a un contagio, a un sida... el círculo mío tampoco era de gamines. Entonces usted nunca va a ver un muchacho gaminoso, oliendo maluco... No le voy a entregar mi bolsa ni mi pitillo, pero es por higiene. Le digo: "ponga la mano y le doy un poquito". Pero a cualquier persona que sea conocida mía, sí, sí le presto la bolsa y hasta el pitillo (entrevista No. 18).

Con mi novio compartí la jeringa muchas veces porque yo sabía quién era él, llevaba cinco años con él, y nosotros nos hemos hecho pruebas del sida y todo. Gracias a Dios todas nos han salido negativas, y también con un amigo (entrevista No. 35).

Por otro lado, también se encontró que en las prácticas de consumo de heroína inhalada o fumada se compartían los utensilios con mucha frecuencia: pitillos ( 9 relatos), papeletas (5), tarjetas o navajas para aspirar la droga (9), el plato para picar (1), el papel de aluminio para fumarla (2) y el tubo para aspirar (1). Así mismo, se detectaron prácticas de compra compartida de la droga y algunas dinámicas de intercambio de las dosis (7).

\section{Sobredosis e intoxicaciones}

Para dar cuenta de la ocurrencia de sobredosis e intoxicaciones entre los consumidores de heroína, se preguntó a los entrevistados si las habían experimentado: 19 (44\%) del total de los 42 entrevistados manifestaron haber tenido síntomas de sobredosis. Entre éstos, 9 manifestaron haber sentido síntomas en una ocasión, 5 en dos, 4 en tres y 1 en cuatro ocasiones. De estos sólo 3 habían sido atendidos en servicios de urgencias. 
Entre los síntomas físicos que los usuarios asociaron con una sobredosis se pueden enumerar: sensación profunda de relajación (24) manifestada como: quedarse dormido, un "relajo" tan grande que no pueden moverse, sueño incontrolable, sentir que el cuerpo se separa del alma y que no se puede mover, falta de coordinación en el habla ("me pesaba la lengua"), pérdida del equilibrio, sensación de embriaguez, mareos, presión baja. Otros síntomas referidos fueron nauseas y vómitos (9), corazón lento (3), rasquiña en la cabeza, los pies y las yemas de los dedos (1), "se me blanqueaban los ojos" (1), flema en la garganta (1), escalofríos (1), asfixia (1), las pupilas se vuelven muy pequeñas (1). Todos estos signos y síntomas coinciden con lo citado en la literatura en relación con la sobredosis ocasionada por la heroína.

Uno de los testimonios dicente con claridad sobre los síntomas de una sobredosis se transcribe a continuación:

Yo sé que me estoy intoxicando porque ya uno se está yendo, ya usted se para y no se siente, como si propiamente el alma saliera del cuerpo, uno siente el cuerpo aquí, y se siente uno aquí y el cuerpo allá y se toca usted el corazón... yo, por ejemplo, me ponía la mano así y era: "pa', pa' ", lento, y muy fuerte (entrevista No. 8).

Relacionando las vías de administración con la ocurrencia de sobredosis, la mayor frecuencia de esta y las sensaciones más fuertes de intoxicación, se manifestaron por los consumidores de heroína intravenosa. Los usuarios de esta sustancia que han tenido síntomas y signos de sobredosis los atribuyen a exceso en el consumo en un mismo momento, uso de mezclas de hroína con otras drogas y heroína "mala" (cortada por los jíbaros con otras sustancias).

Llama la atención entre los entrevistados que manifestaron haber tenido síntomas de una sobredosis, las prácticas que utilizaban para evitar la muerte:

"moverse y caminar constantemente y como sea para no quedarse dormidos, y consumir en grupos para asegurar el cuidado de los otros" (testimonio informante 25).

Finalmente, y por la importancia que tiene la sobredosis para la salud pública, en relación con la pérdida de años de vida por muerte y costos para el sistema de salud, es necesario dar cuenta en esta investigación de lo manifestado por un número importante de los entrevistados (10) que afirmaron que en sus grupos de consumidores, observaron con frecuencia casos de sobredosis que ameritaron llevar al consumidor a un servicio de urgencias. No se obtuvieron datos de la suerte de estos adictos a la heroína.

\section{Ideas e intentos suicidas entre los consumidores de heroína}

Un poco más de la mitad (24) de los consumidores entrevistados han tenido ideas suicidas y en dos casos intentos reales de suicidio. Las ideas que manifiestaron están vinculadas con las prácticas del consumo y, sobre todo, relacionadas con las depresiones posconsumo y la angustia que les genera el no poder parar de consumir la sustancia.

Las ideas suicidas están relacionadas con sobredosis inducidas con heroína (8), sobredosis inducidas con otras drogas (5), cortarse las venas (4), desear que lo asesinen (1), pensar en tirarse de un balcón (1), ponerse una pistola en la cabeza (1), darse golpes en la cabeza (1) o propiciar accidentes (1). No se encontraron en ellos ideas muy elaboradas al respecto. Los dos casos hallados con intento suicida, 
lo hicieron, uno induciéndose una sobredosis de heroína y el otro intentando provocar un accidente mientras conducía una motocicleta.

\section{Relaciones sexuales y uso de condón}

Al respecto, todos los entrevistados (exceptuando un caso) declararon que han tenido relaciones sexuales bajo los efectos de la heroína. Casi todos afirmaron que gracias a la droga adquirieron más control y un retraso evidente en la eyaculación. También se detectó en las respuestas, que existe, entre los entrevistados, un alto nivel de promiscuidad sexual.

[...] Me gustaba mucho tener relaciones sexuales bajo efectos de heroína, porque, la heroína es como un estimulante también, lo retarda mucho a uno, lo pone más activo. Sí, la verdad es que yo sin heroína ya no tenía relaciones sexuales (entrevista No. 5).

Frente al uso de protección, de los 41 usuarios entrevistados que habían tenido relaciones sexuales, el $53 \%$ usaba condón, el $17 \%$ algunas veces y el $30 \%$ no se protegía.

\section{Delitos relacionados con el consumo}

Entre las respuestas de los entrevistados, se encontró que el 78,5\% (33) ha robado para consumir y 12 de ellos $(28,5 \%)$ en ocasiones han traficado con drogas. De estos ninguno fue sometido a juicio porque, en su gran mayoría, robaron a las familias o a amigos, y no fueron denunciados.

También se encontró, aunque en un pequeño porcentaje, 4,7\%, que 2 consumidores de heroína han herido o intentando herir a otros y esto ha ocurrido bajo efectos del consumo y, sobre todo, está relacionado con la mezcla de drogas.

Una vez mezclé pepas y heroína. Estaba en un apartamento con un amigo y de un momento a otro me inyecté y me tomé la pepa y se me metió en la película que el hombre me iba a violar, quebré una botella de cristal de esas que son como bomboneras y le metí ocho puñaladas (entrevista No. 40).

Una vez le apunté con un arma a un parcero, le dije que lo iba a quemar. Le bajé el seguro, le bajé el tramo al gatillo, y le dije: "iTe voy a matar pirobo!". "¿Por qué me vas a matar?" "¿Por qué te voy a matar? Porque me da la gana de matarte". Un parcero que me vio por detrás, me dijo: "¿Plaga, qué te pasa?" "Yo no sé". "Quieto, quieto", me dijo, y le bajé el tramo y el seguro al revolver. Entonces yo me pregunté: "¿Qué me pasa? No sé, no sé qué pasa". Casi lo mato (entrevista No. 25).

\section{Problemas de salud}

Con respecto a los problemas de salud ocasionados por el consumo, $33(78,5 \%)$ entrevistados, afirmaron haber padecido algún síntoma o enfermedad relacionada con el consumo de heroína, entre ellos trastornos alimentarios (pérdida gradual del apetito, pérdida de peso, estreñimiento), insomnio, sensación de cansancio, opresión en el pecho, fibrosis en las venas, dificultades visuales y temblor en las manos. Se quejaron, además, de trastornos en la temperatura corporal y manifestaron que se mantienen muy fríos. 
Muchos de estos síntomas son ocasionados por las propiedades toxifarmacológicas de los opiáceos, y la fibrosis en las venas se debe a los repetidos traumas que los inyectores producen en los sitios de punción. En relación con las enfermedades infectocontagiosas, solo un consumidor que usaba la droga por vía intravenosa reconoció tener hepatitis $\mathrm{C}$.

\section{Realización de tareas bajo efecto de drogas}

Conducir un vehículo, desde un auto, una motocicleta, hasta una bicicleta, bajo el efecto de la heroína ha sido la constante en 25 (59,5\%) de los entrevistados de esta muestra. De estos, 10 manifestaron haber tenido accidentes y 1 explicó que manejaba un taxi de servicio público cuando ocurrió un suceso en el que se vio involucrado uno de sus pasajeros.

\section{DISCUSIÓN}

La morbilidad y mortalidad, asociada al consumo de heroína se ha mantenido estable. Un ejemplo lo constituye España, allí la máxima incidencia de uso de heroína se alcanzó en la primera mitad de los ochenta, ${ }^{23}$ el mayor impacto y visibilidad de la epidemia de VIH se produce a principios de los noventa y la mortalidad relacionada con las drogas alcanza entonces su punto crítico, llegando a ser la primera causa de muerte entre los jóvenes de las grandes ciudades. ${ }^{24,25} \mathrm{~A}$ partir de los datos publicados, ${ }^{26,27}$ se estima que en España el mayor impacto de la mortalidad por sobredosis se produce entre 1991 y 1992, con más de 1700 muertes anuales (11,5 muertes por cada 100000 jóvenes entre 15 y 39 años, 10,1 $\%$ de todas las muertes en esas edades), en más del $90 \%$ de las cuales estaba implicada la inyección de heroína. Los nuevos diagnósticos de sida ligados a inyección de drogas alcanzan su máximo nivel entre 1993 y 1995 con más de 3500 casos anuales, y la mortalidad por VIH entre 1995 y 1996 con casi 4300 muertes anuales (27,4 por cada 100000 jóvenes entre 15 y 39 años, 25,3\% de todas las muertes en esas edades). La máxima incidencia de VIH ligado a inyección de drogas se produce probablemente entre 1985 y $1987,{ }^{19}$ con aproximadamente 14 500 infecciones anuales. ${ }^{20}$

El perfil sociodemográfico de los consumidores de heroína, en Medellín y el Valle de Aburrá, tuvo gran similitud en cuanto al género con investigaciones realizadas en otras latitudes, donde son predominantes los consumidores hombres solteros y menores de 20 años, aunque se diferenció de algunos estudios en la edad y la formación académica. Por ejemplo, en una iniciativa similar de Sánchez y Berjano, ${ }^{28}$ en España, se encuentra que igualmente los sujetos adictos son predominantemente varones, solteros, de unos 26 años, sin hijos; se diferencian en el grado de escolaridad, en tanto tienen mayoritariamente estudios primarios sin finalizar; y en edad, puesto que en la muestra de este estudio, la edad era significativamente más baja. Resultados similares a este estudio español sobre datos sociodemográficos de consumidores de heroína son informados por el Plan Nacional sobre Drogas de España en el año 1997. ${ }^{29}$

En relación con la edad, este estudio detectó consumo en edades más tempranas a las registradas por otros trabajos, como ya se dijo arriba. Autores como Amodia, ${ }^{30}$ Comas y otros, ${ }^{31}$ Marina, ${ }^{32}$ Plan Regional de Drogas, ${ }^{33}$ Ruíz, Cervera, Gutiérrez, San Narciso, Olmos-Espinosa, y otros, ${ }^{34-38}$ informan en sus trabajos una mayor prevalencia de consumo de opiáceos en edades comprendidas entre los 25 y los 29 años, con predominio en el sexo masculino, solteros o separados, con baja 
calificación académica, desempleados y que consumen habitualmente otras drogas de abuso, como cocaína, benzodiacepinas o alcohol.

En el estudio de Sánchez y Berjano, ${ }^{28}$ en cuanto a lo que interesa a la presente investigación, ambas poblaciones, la española y la colombiana, se iniciaron en el consumo del tabaco y alcohol a temprana edad, con historia toxicológica de consumo de diferentes sustancias psicoactivas: marihuana, alucinógenos, anfetaminas, benzodiazepinas y cocaína, hasta que finalmente, a la edad aproximada de 19 años, comenzaron el consumo de heroína. Esto es similar a lo hallado en la presente investigación, donde la mayoría de los participantes comenzaron a usar heroína entre los 17 y los 20 años. De la Fuente De Hozk y otros, ${ }^{39}$ en el marco del proyecto ITINERE, también registran como edad de inicio en el consumo de heroína, por la vía fumada o inhalada, los 18 años.

Con respecto al policonsumo, en dos estudios, ${ }^{40,30}$ los adictos empiezan a consumir heroína después de haber incursionado en el uso de otras sustancias. En esencia, el heroinómano está predispuesto y apto para ser un policonsumidor, es decir, que puede llegar a utilizar toda clase de drogas. Según Draper, ${ }^{41}$ este termina consumiendo de manera simultánea toda la gama de tóxicos, buscando evitar el síndrome de abstinencia a la heroína que le resulta muy molesto y le causa mucho miedo.

Preocupa en este estudio, la temprana edad en el inicio del consumo de esta sustancia, lo que implica una mayor carga de morbilidad y unos mayores costos para el sistema de salud en una franja poblacional, la de los jóvenes, que se caracteriza por ser más bien saludable.

En cuanto a la población femenina participante en este estudio, 4 de las 8 mujeres afirmaron ser policonsumidoras, presuntamente debido a la influencia de sus parejas o de amigos consumidores, y a tener facilidad para obtener estas drogas. El consumo lo hacen, por lo general, en compañía, utilizando comúnmente los métodos de inhalación e inyección. Estos hallazgos son similares a lo encontrado por Meneses ${ }^{42}$ en un estudio etnográfico de mujeres consumidoras de heroína, donde se notifica que las 25 participantes mostraron algunos factores que influían en el inicio de su consumo, tales como un consumo previo de otras drogas, la familiarización con la sustancia, y la influencia del entorno, en tanto ellas habían observado el consumo en personas cercanas. La vía de uso más común es la esnifada o fumada. Las consumidoras son iniciadas por otras personas, entre las que se encuentran sus parejas, amigos y diversos familiares.

Con respecto a la historia familiar de consumo, tanto en el estudio de Sánchez y Berjano, ${ }^{28}$ como en este, los heroinómanos informaron estar viviendo con algún familiar con problemas de drogodependencia. En esta misma dirección y recordando los aportes de Volkow y Li (2004, citados por Rodríguez, 2006),43 la adicción a la heroína supone un contacto crónico con ambientes familiares y genéticos, así como hereditarios. En este estudio, casi la totalidad de los participantes, $88 \%$, tenía antecedentes familiares y parentales en el consumo de drogas, que incluía a abuelos, tíos, primos, padres y hermanos, quienes consumían tabaco, alcohol, marihuana, y cocaína.

De otro lado, y en cuanto al tipo y calidad de la heroína consumida, a las vías de administración, a la dosis y frecuencia de consumo, se observó que en el $70 \%$ de los consumidores (29), la cantidad de heroína consumida no superó $1 \mathrm{~g} /$ día, lo cual podría tener dos explicaciones: 1. Los usuarios llevaban poco tiempo en el consumo y, por tanto, aún no habían desarrollado tolerancia. 2 . La heroína que consumían es 
de buena calidad, lo que hace que el consumidor requiera dosis más bajas durante el día.

El patrón que une la cantidad de dosis consumida con la cantidad de compra y la frecuencia de consumo varía considerablemente, debido a los siguientes factores: vía de administración, dinero que se tiene y calidad de la heroína. Se puede decir que los consumidores que inhalan consumen mayor número de dosis al día y con más frecuencia. A mayor dinero, mayores posibilidades de comprar varios gramos para varios días, y la calidad de la heroína puede explicar en parte, no solo un efecto más prolongado $y$, por tanto, menos frecuencia en el consumo, sino también la preferencia por una u otra vía de administración. Elzo, Laespada y Vielva, ${ }^{44}$ refieren que existen varias razones para explicar cambios en las vías de consumo. Una de ellas, al parecer la más ampliamente aceptada, se relaciona con el tipo de heroína que circula en la calle. La heroína marrón es menos soluble que la blanca, razón por la que su consumo se realiza a través de su combustión y posterior inhalación; la blanca sería más idónea para su administración por vía parenteral.

En Medellín y su área metropolitana, al parecer, circulan los tres tipos de heroína (marrón, blanca y negra), siendo la más disponible la marrón, también conocida como brown sugar, que habitualmente es usada para fumar, pero que es utilizada por los consumidores entrevistados por cualquier vía, no exentos de las dificultades para diluirla cuando su consumo es por vía parenteral y, seguramente, con pobre absorción por vía nasal. La heroína blanca, por su alto costo, es menos usada y está menos disponible en el mercado. Cuando sale a la calle, es también la más adulterada. Todos estos tipos de heroína, al parecer, son colombianas, pues no existen evidencias de ser parte de las rutas de narcotraficantes asiáticos o mexicanos.

La heroína marrón denominada No.3, parece ser el tipo de esta droga más común en el mercado al que tienen acceso los consumidores entrevistados. Originalmente procedía del Triángulo de Oro conformado por Laos, el norte de Tailandia y el noroeste de Birmania. Pero en Colombia no proviene de esas regiones, sino que es producida en el país. Su aspecto es terroso y granulado, de color gris sucio, marrón, terracota, de textura parecida a la del yeso de construcción. Su contenido de heroína oscila entre el 25 y el $50 \%$ y está destinada generalmente a ser fumada. Este tipo de heroína, también producida en México y llamada Tecata, se mercadea ordinariamente en la costa oeste norteamericana.

También hay referencias a la heroína No. 4, conocida también con el nombre de tailandesa o china. Es la más refinada y su contenido de heroína puede alcanzar el $90 \%$ antes que la sustancia sea manipulada. Es de color blanco crema o amarilloso, de consistencia fina y esponjosa. Su textura es la de la leche en polvo mezclada con algo de azúcar y está destinada, indistintamente, a ser fumada o inyectada por vía intravenosa o subcutánea.

En la información obtenida, hay mención también a una heroína que Gómez ${ }^{22}$ ha denominado Black Tar, un tipo muy común en el mercado estadounidense, en especial en la costa oeste, con una apariencia similar a la brea o a las rocas de carbón, de un color café muy oscuro, casi negro. Esta heroína procedente de México tiene un proceso deficiente y burdo, es frecuentemente cortada (mezclada) y se vende para inyectar.

De acuerdo con la vía de administración, en este estudio, de la muestra de 42 usuarios, $32(76 \%)$ no se inyectaban y $10(23 \%)$ sí lo hacían, pero todos alternaban o han alternado las vías de consumo inhalada o fumada. Estos resultados son distintos a los de otros estudios, entre ellos el realizado por March 
Cerdà y otros, ${ }^{45}$ quienes encuentran que entre los participantes que se inician en el consumo por la vía pulmonar, un pequeño número alterna con la vía intravenosa, y la gran mayoría no cambia de vía. Afirman estos autores que quienes se inician en el consumo de heroína inhalada, en general continúan con esa forma de consumo y aunque en ciertos trabajos se ha planteado que la vía pulmonar es una puerta para el consumo de heroína por vía intravenosa, ${ }^{46}$ otros establecen que los usuarios mantienen esta vía sin pasarse a la inyectada. ${ }^{47} \mathrm{En}$ los resultados mostrados, la gran mayoría de usuarios se inició con la vía inhalada.

March Cerdà y otros, ${ }^{45}$ afirman que, en general, las explicaciones expuestas para el paso hacia el uso de la vía inyectada desde la pulmonar son, la presión social, tener una pareja que se inyecta, la influencia del mercado y los cambios en las costumbres, entre otras. ${ }^{48,49}$ También se ha sugerido que una mayor frecuencia en el consumo por vía pulmonar, indicador de mayor dependencia, podría estar asociada al cambio hacia la vía inyectada por considerarse esta más eficaz y efectiva. ${ }^{50}$ Estas mismas argumentaciones fueron dadas por los usuarios de heroína de este estudio. En concordancia con otros estudios, no se han observado transiciones inversas, es decir, de usuarios por vía intravenosa a la vía pulmonar, igual que en el estudio referido por Swift, Maher, Sunjic, ${ }^{51}$ dado que ninguno de los participantes que se iniciaron con la vía intravenosa ha dejado de inyectarse, si bien algunos tienden a alternar las vías.

Los consumidores entrevistados calificaron la preferencia por otras vías frente a la vía intravenosa como una ruta de administración de menor riesgo, y no solo en lo que al contagio de VIH se refiere, al igual que lo encontrado en otros estudios ( $D e$ la Fuente, Bravo, Lew, Barrio, Soriano, Royuela; Latkin, Knowlton, Sherman), ${ }^{52,53}$ sino también con otros aspectos relacionados con la salud, como el deterioro de las venas o infecciones relacionadas con la venopunción. Así mismo, los estudios indican que el consumo de heroína no inyectada conlleva un riesgo menor de sobredosis, ${ }^{54,55}$ y puede asociarse a una menor dependencia, ${ }^{56}$ dato que también aportó este estudio.

Según March Cerdà y otros, ${ }^{45}$ los factores que mejor explican la probabilidad de inyectarse, son el sexo, la edad, la edad de inicio en el consumo y el número de tratamientos con metadona realizados con anterioridad. En el estudio presentado se conocieron las tres primeras variables. Las mujeres que han participado en el estudio español, así como las entrevistadas, prefirieron la vía pulmonar, lo cual se ha indicado también en otros trabajos. ${ }^{57}$ Por otro lado, los más jóvenes, así como quienes se han iniciado más tardíamente en el consumo de heroína, tienden a usar la vía inyectada en menor medida. La edad de inicio en el consumo, como factor de riesgo, es una constante en los estudios sobre drogas, donde se indica que cuánto más jóvenes comienzan las personas a consumir, mayores son los daños asociados. $^{58}$

En otros trabajos se puede observar edades tempranas de inicio en el uso de heroína en grupos que consumen preferentemente por la vía intravenosa, ${ }^{59}$ así como una mayor inclinación por parte de los usuarios más jóvenes hacia la vía pulmonar. ${ }^{60}$ El consumo por la vía pulmonar podría estar acercando la heroína a poblaciones jóvenes, ${ }^{61}$ posiblemente por su rápida biodisponibilidad, con la ventaja de poder administrarse sin necesidad de utilizar una aguja. ${ }^{62}$ Todo esto es posible que esté sucediendo en el medio colombiano por la temprana edad de inicio en el consumo de esta sustancia que están teniendo los jóvenes en Medellín y su área metropolitana, que, como ya se ha dicho, suele fluctuar entre los 17 y los 20 años.

Es previsible de todas maneras, como se ha visto en estudios previos (De la Fuente, Barrio, Royuela, Bravo, ${ }^{63}$ De la Fuente, Brugal Puig, Ballesta Gómez, Bravo Portela 
y otros $\left.{ }^{39}\right)$, que la vía de uso de la heroína pueda cambiar a lo largo de la carrera de un consumidor, y no siempre hacia la inyección, aunque esta sea considerada la vía más eficiente en términos de efecto/coste.

Las sobredosis e intoxicaciones constituyen también problemas importantes para la salud pública por la morbilidad y mortalidad que ocasionan. El 44\%, 19 de los 42 entrevistados, manifestó haber tenido síntomas de sobredosis. Una cifra alta para lo pequeño del número y que augura problemas serios en el futuro, pues en España, según afirman De la Fuente y otros ${ }^{20}$ la mortalidad por sobredosis ha sido muy alta (más de 700 muertes anuales), agregan que entre los consumidores por vía intravenosa persiste una elevada prevalencia de VIH y hepatitis C, y están emergiendo consecuencias relacionadas con hepatopatías crónicas.

Llama la atención, que aunque es común pensar que los consumidores de drogas no cuidan su salud y que son unos irresponsables, "que se están matando poco a poco", nada más lejos de la realidad. Los usuarios de drogas aprenden a conocer la sustancia, los riesgos de los consumos; aprenden, aunque con excepciones por causa de la aparición de riesgos no previstos, a manejar la sustancia y a saber cuando están teniendo signos y síntomas de una intoxicación. Al menos esto fue lo que se demostró en este trabajo: los consumidores aprenden a reconocer cuándo no deben consumir más heroína y desarrollan técnicas para evitar complicaciones, como luchar para no quedarse dormidos.

De otro lado, y en relación con ideas y gestos suicidas entre los consumidores de heroína, hecho que también preocupa a la salud pública por la carga que implica la pérdida en años de vida para un sector tan joven de la población, se destaca en la muestra examinada, el alto índice de ideas suicidas que se presentaron entre los consumidores de heroína; aunque, por fortuna, sin llegar al acto y tener éxito, pero ello sí constituye una llamada de advertencia para los responsables de la salud pública, acerca de la importancia de prevenir los consumos de esta droga entre los jóvenes y ofrecer tratamiento y rehabilitación a los que ya tienen este problema. Coinciden estos rasgos con algunas de las características de personalidad del grupo de consumidores encontradas por Sánchez y Berjano ${ }^{28}$ y García Rodríguez y otros, en una muestra de consumidores españoles. ${ }^{64}$

Otro aspecto que afecta la salud de los consumidores de drogas está relacionado con las enfermedades de transmisión sexual (ETS) adquiridas por prácticas sexuales sin protección. En este estudio no usaba protección un gran porcentaje de los consumidores entrevistados ( $30 \%$ no lo hace y el $17 \%$ sólo ocasionalmente se protege). Esto, sumado a la promiscuidad, constituye un grave riesgo para la salud pública, por la posibilidad de transmisión de enfermedades venéreas que terminan afectando a otros consumidores y a sus parejas. Si a ello se le agrega que muchos de estos consumidores usan la vía intravenosa y comparten jeringas, los riesgos de transmisión de $\mathrm{VIH} /$ sida y hepatitis $\mathrm{B}$ y $\mathrm{C}$, no solo por compartir la jeringa, sino por la transmisión vía sexual, son altos para la población de consumidores y su red más cercana.

VIH/sida, hepatitis B y C son otras de las enfermedades susceptibles de ser padecidas por los consumidores de heroína, con altos costos para la salud pública, particularmente cuando la vía de administración es la intravenosa, en relación con malas prácticas de consumo por el uso compartido de jeringas, su reutilización y el compartir la parafernalia y los pitillos. Muchas de estas prácticas se demostraron en este estudio, pero ninguno de los entrevistados manifestó estar infectado por el virus de la inmunodeficiencia humana, ni tener hepatitis B. Sólo uno de los participantes en el estudio afirmó padecer hepatitis C. Por tratarse de una investigación cualitativa, que no aplicó pruebas serológicas, y ante la falta de 
protocolos de atención claros en los centros de tratamiento para los dependientes de esta sustancia, que no tienen como regla la práctica de estos exámenes, los números registrados en este estudio acerca de este punto, no pueden considerarse confiables y podría hipotéticamente decirse que el número de infectados puede ser mucho mayor, pues son numerosos los estudios que muestran esta asociación, ${ }^{40,65-}$ ${ }^{69}$ más cuando se encontraron evidencias de malas prácticas de consumo y de intercambio de accesorios y jeringas entre consumidores.

Por otro lado, es bien sabido el riesgo de infecciones locales en los sitios de inyección, también por malas prácticas como no limpiar la zona donde se va a inyectar, contaminación de los utensilios o sistémicas -septicemias-, ocasionadas por la contaminación de la sustancia. Se han descrito casos de heroína contaminada con esporas de hongos. Uno de los entrevistados, al respecto, comentó esta experiencia, que aunque anecdótica, hace reflexionar sobre otro riesgo importante para la salud de los consumidores de esta sustancia psicoactiva:

Sí se me ha afectado la salud y a veces por contaminantes. Les voy a explicar esto: el algodón es para filtrar la droga y que no surja ningún pelo o ninguna otra bacteria y todo lo que uno se inyecta sea lo más puro; hay veces que uno se inyectaba puede ser hasta un pedacito de tierra o un pelo y eso entrar en las venas y le causa a uno muchos escalofríos y un descontrol de fiebre. Uno no puede controlar eso, y ahí es cuando uno sabe que uno se inyectó algo fuera de lo común (entrevista No. 28).

La realización de tareas bajo el efecto de drogas fue otra de las categorías analizadas. Se encontró en este estudio riesgos importantes de accidentalidad al conducir o realizar tareas en estas condiciones, lo que tiene también implicaciones importantes para la salud pública porque no solamente se puede lastimar el individuo que consume sino también afectar a terceros.

Se tienen datos de consumos de esta sustancia en zonas como los Santanderes, Cundinamarca, Valle del Cauca, Cauca y Eje Cafetero. Ello amerita que se conozcan sus particularidades con el fin de hacer óptimas las intervenciones, pues estudios como los realizados por De la Fuente y otros, ${ }^{39}$ demuestran que hay diferencias en las prácticas de consumo en un mismo país.

El consumo de heroína no sólo es una realidad emergente en Colombia, sino que, según lo encontrado en la investigación, acecha de manera importante a la salud pública. Se hace urgente realizar estudios específicos de prevalencia e incidencia de esta sustancia que muestren la realidad de una posible pandemia en el país, y realizar estudios cualitativos más amplios para dar cuenta de las características del consumo y de los consumidores en las distintas regiones colombianas.

\section{REFERENCIAS BIBLIOGRÁFICAS}

1. Observatorio de Drogas de Colombia [sitio en Internet]. [citado Mar 2009].

Disponible en: http://odc.dne.gov.co/spip.php?rubrique9

2. Segundo Estudio de Consumo de Sustancias Psicoactivas en la Ciudad de Santa Fe de Bogotá. Alcaldía Mayor de Santafé de Bogotá. Unidad Coordinadora de Prevención Integral (UCPI). Santa Fe de Bogotá: Universidad de los Andes; 1992. 
3. Ministerio de Salud de Colombia. Estudio Nacional de salud Mental y Consumo de Sustancias Psicoactivas. Santa Fé de Bogotá: Oficina de Comunicaciones; 1994.

4. Estudio Nacional sobre Consumo de Sustancias Psicoactivas en Colombia. 1996. Dirección Nacional de Estupefacientes. Bogotá D.C. [sitio en Internet]. 1996 [citado 18 Feb 2008]. Disponible en: http://www.dne.gov.co/?idcategoria $=845$

5. Ministerio de Salud de Colombia. Segundo Estudio Nacional de salud Mental y Consumo de Sustancias Psicoactivas. Versión preliminar. Santa Fe de Bogotá: Oficina de Comunicaciones; 1998.

6. Sondeo Nacional sobre Consumo de Drogas en Jóvenes. Programa Presidencia RUMBOS. Presidencia de la República de Colombia. Bogotá D.C.: Comisión Nacional de Investigación sobre Drogas; 1999.

7. Sistema de Vigilancia Epidemiológica del Consumo de Sustancias PsicoactivasVESPA- Formato para Centros Penitenciarios. Gobernación de Caldas. Manizales: Unidad de Prevención Integral del Departamento de Caldas; 1999.

8. VESPA UNIVERSITARIO. Sistema de Vigilancia Epidemiológica sobre Consumo de Sustancias Psicoactivas en Población Universitaria. Fundación Universitaria Luis Amigó. Red Universitaria en Farmacodependencia. RED UNIR. Secretaría de Salud de Medellín. Medellín: Fundación Universitaria Luis Amigó; 2000.

9. Comisión Nacional de Investigación en Drogas. (2001). Encuesta Nacional sobre Consumo de Sustancias Psicoactivas en jóvenes de 10 a 24 años [sitio en Internet]. [citado 22 Mar 2008]. Disponible en:

http://sparta.javeriana.edu.co/psicologia/publicaciones/actualizarrevista/archivos/V 5N306 consumodrogas.pdf

10. Mejia IE, Perez A. La inyección de drogas en Bogotá: una amenaza creciente. Rev Adicciones. 2005;17(3): 251-60.

11. Evaluación del Progreso de Control de Drogas. 2005-2006. Colombia. MEM. CICAD/OEA [sitio en Internet]. [citado 30 Sept 2008]. Disponible en: http://www.cicad.oas.org/MEM/esp/Informes/Cuarta\%20Ronda\%20Full/Colombia\% 20-\%20Fourth\%20Round\%20-\%20ESP.pdf

12. Álvarez Gómez M, Suárez Bermúdez AM, Giraldo Ferrer LF, Londoño Restrepo J, Hoyos Arboleda TM, Bedoya Marín DC, et al. Características de las personas adictas a la heroína en tratamiento en una empresa social del Estado de enero de 2003 a junio de 2008. SMAD [serie en Internet]. 2009 [citado 30 Sept 2008];5(1). Disponible en:

http://redalyc.uaemex.mx/src/inicio/IndArtRev.jsp?iCveNumRev=13061\&iCveEntRe $\underline{v=803}$

13. Estudio Nacional sobre Consumo de Sustancias Psicoactivas en Colombia. 2008. Ministerio de la Protección Social, Dirección Nacional de Estupefacientes [sitio en Internet]. [citado 25 Mar 2009]. Disponible en: http://odc.dne.gov.co/IMG/pdf/20.pdf 
14. Chitwood DD, Sánchez J, Comerford M, McCoy CB. Primary preventive health care among injection drug users, other sustained drug users, and non-users. Subst Use Misuse. 2001;36(6-7):807-24.

15. Brugal MT, Barrio G, Royuela L, Bravo MJ, De la Fuente L, Regidor E. Estimación de la mortalidad atribuible al consumo de drogas ilegales en España. Med Clin (Barc). 2004;123:775-7.

16. Castilla J, De la Fuente L. Evolución del número de personas infectadas por el virus de la inmunodeficiencia humana y de los casos de sida en España: 19801998. Med Clin (Barc). 2000;115:85-9.

17. Centro Nacional de Epidemiología. Instituto de Salud Carlos III. Sistema de Información sobre nuevos diagnósticos de VIH autonómicos. Vigilancia epidemiológica de VIH en España. Valoración de la epidemia de VIH en España a partir de los sistemas de notificación de casos de las comunidades autónomas [sitio en Internet]. [citado 20 Jun 2008]. Disponible en:

http://www.isciii.es/htdocs/pdf/nuevos diagnosticos ccaa.pdf

18. Delegación del Gobierno para el Plan Nacional sobre Drogas. Observatorio Español. Plan Nacional sobre Drogas. Indicadores tratamiento, urgencias y mortalidad. Informe año 2002. Madrid: Ministerio de Sanidad y Consumo [sitio en Internet]. 2006 [citado 21 jun 2008]. Disponible en: http://www.pnsd.msc.es/Categoria2/observa/seipad/indicad 2002.htm

19. Brugal MT, Queralt A, Graugés D, García V, Vecino C. Sistema d'Informació de Drogodependències de Barcelona. Actualització dels indicadors de drogues corresponent al $2 n$ trimestre del 2005. Barcelona: Agència de Salut Pública de Barcelona; 2005.

20. De la Fuente L, Brugal MT, Domingo-Salvany A, Neira- Leon M, Barrio G. Más de treinta años de drogas ilegales en España: una amarga historia con algunos consejos para el futuro. Rev Esp Salud Pública. 2006;80(5).

21. Stockley D. Drogas. Bilbao: Edex Kolektiboa; 1995.

22. Gómez L. Cartel, historia de la droga. Santafé de Bogotá: Grupo Editorial Investigación y Concepto; 1991.

23. Sánchez Niubó A, Domingo-Salvany A, Gómez J, Brugal MT. Estimación de la incidencia de consumo de drogas. Gac Sanit. 2005;19(Supl. 1):98.

24. De la Fuente L, Barrio G, Vicente J, Bravo MJ, Santacreu J. The impact of drugrelated deaths on mortality among young adults in Madrid. Am J Public Health. 1995;85(1):102-5.

25. Ortí RM, Domingo-Salvany A, Muñoz A, Macfarlane D, Suelves JM, Antó JM. Mortality trends in a cohort of opiate addicts, Catalonia, Spain. Int J Epidemiol. $1996 ; 25: 545-53$.

26. Brugal MT, Barrio G, Regidor E, Mestres M, Caylà JA, De la Fuente L. Discrepancias en el número de muertes por reacción aguda a sustancias psicoactivas registradas en España. Gac Sanit. 1999;13:82-7. 
27. Delegación del Gobierno para el Plan Nacional sobre Drogas. Observatorio Español sobre Drogas (OED). Informe 2004. Madrid: Ministerio de Sanidad y Consumo; 2005.

28. Sánchez H, Berjano P. (1996). Características de personalidad en sujetos drogodependientes. Comunidad Terapéutica "Balsa Blanca" (Valencia) y Facultad de Psicología de Valencia [sitio en Internet]. [citado 3 Feb 2008]. Disponible en: http://www.psicothema.com/pdf/44.pdf

29. Observatorio Español sobre Drogas. Informe $\mathrm{N} .^{\circ}$ 1. Ministerio del Interior Delegación del Gobierno para el Plan Nacional sobre Drogas. Madrid: Secretaría General Técnica; 1997.

30. Amodia Díez R. Epidemiología de las toxicomanías en la Red de Salud Mental de Asturias (1980-1989). Cuadernos Asturianos de Salud. Oviedo: Servicio de Publicaciones del Principado de Asturias; 1992.

31. Comas D, Carrón J, Hernández MI. Metodología de los estudios de seguimiento. Resultados de una investigación. Adicciones. 1992;4 (2):111-26.

32. Marina P. Adictos a la heroína en Asturias. Un estudio de seguimiento. En: Bobs J, González-Quiroz P, González MP, editores. 30 años de alcohol y otras drogas en Asturias. Oviedo: Gofer; 1996.

33. Plan Regional sobre Drogas. Memoria 1994. Oviedo: Servicio de Publicaciones del Principado de Asturias, 1995.

34. Ruíz R, Casete L, Carrera IH. Estudio preliminar sobre la incidencia y valor pronóstico del codiagnóstico psiquiátrico en usuarios de una Comunidad Terapéutica. Oviedo: Socidrogalcohol; 1996.

35. Cervera G, Valderrama JC, Bolinches F. Pauta de desintoxicación frente a estabilización y mantenimiento con metadona en adictos a opiáceos con trastorno de personalidad. Psiquiatría Biológica. 1997;4(5):181-6.

36. Gutiérrez E, Sáiz PA, González MP. Definición del perfil de buen respondedor a los programas de mantenimiento con naltrexona. Adicciones. 1998;10(4):321-33.

37. San Narciso GI, Carreño JE, Pérez SF. Evolución de los trastornos de personalidad evaluados mediante el IPDE en una muestra de pacientes heroinómanos en tratamiento con naltrexona. Adicciones. 1998;10(1):7-22.

38. Olmos-Espinosa R, Madoz-Gúrpide A, Ochoa E. Situación al año de los adictos a opiáceos que siguieron tratamiento ambulatorio tras desintoxicación hospitalaria. Adicciones. 2001;13(2):173-8.

39. De la Fuente De Hoz L, Brugal Puig MT, Ballesta Gómez R, Bravo Portela MJ, Barrio Anta G, Domingo Salvany A, et al. Metodología del estudio de cohortes del proyecto ITINERE sobre consumidores de heroína, en tres ciudades españolas y características básicas de los participantes. Rev Esp Salud Pública. 2005;79(4):47591.

40. García López A, Ezquiaga E. Estudio descriptivo de 433 pacientes que acudieron a un centro de salud mental. Adicciones. 1991;3(2):167-80. 
41. Draper R. Yo me drogo, tú te drogas, él se droga... Barcelona: Plaza \& Janes Editores; 1986.

42. Meneses Falcón C. Mujer y heroína. Proyecto Hombre. 1997; (23):10-5.

43. Rodríguez G. Sistemas cannabinoide y purinérgico: posibles sustratos neurobiológicos de la drogadicción. Universidad Pompeu Fabra. Departamento de ciencias experimentales y de la salud. Laboratorio de neurofarmacología. Barcelona. 2006 [sitio en Internet]. [citado 2 Mar 2008]. Disponible en:

http://www.tdx.cat/TDX-0710107-140631

44. Elzo J, Laespada MT, Vielva I. Informe 2 del Observatorio Vasco de Drogodependencias. Vitoria-Gasteiz: Servicio Central de Publicaciones del Gobierno Vasco; 1999.

45. March Cerdà JC, Oviedo-Joekes E, Romero Vallecillos M, Sánchez-Cantalejo E. Factores asociados al uso de la vía pulmonar e intravenosa en una muestra de consumidores en Granada. Rev Esp Salud Pública. 2005;79(3).

46. Strang J, Griffiths P, Powis B, Abbey J, Gossop M. How constant is an individual's route of heroin administration? Data from treatment and non-treatment samples. Drug Alcohol Depend. 1997;46(1-2):115-8.

47. Griffiths $P$, Gossop M, Powis B, Strang J. Transitions in patterns of heroin administration: a study of heroin chasers and heroin injectors. Addiction. 1994;89(3):301-9.

48. Strang J, Griffiths P, Powis B, Gossop M. Heroin chasers and heroin injectors: differences observed in a community sample in London, UK. Am J Addict $.1999 ; 8(2): 148-60$.

49. Van Ameijden EJ, Coutinho RA. Large decline in injecting drug use in Amsterdam, 1986-1998: explanatory mechanisms and determinants of injecting transitions. J Epidemiol Comm Health. 2001;55(5):356-63.

50. Bravo MJ, Barrio G, de la Fuente L, Royuela L, Domingo L, Silva T. Reasons for selecting an initial route of heroin administration and for subsequent transitions during a severe HIV epidemic. Addiction. 2003;98(6):749-60.

51. Swift W, Maher L, Sunjic S. Transitions between routes of heroin administration: a study of Caucasian and Indochinese heroin users in south-western Sydney, Australia. Addiction. 1999; 94(1):71-82.

52. De la Fuente L, Bravo MJ, Lew C, Barrio G, Soriano V, Royuela L. Prevalencia de infección por el virus de la inmunodeficiencia humana y de conductas de riesgo entre los consumidores de heroína de Barcelona, Madrid y Sevilla: un ejemplo de las ventajas de centrar los estudios en los consumidores y no sólo en los usuarios por vía intravenosa. Med Clin (Barc). 1999; 113(17):646-51.

53. Latkin CA, Knowlton AR, Sherman S. Routes of drug administration, differential affiliation, and lifestyle stability among cocaine and opiate users: implications to HIV prevention. J Subst Abuse. 2001;13(1-2):89-102.

54. Darke S, Ross J. Fatal heroin overdoses resulting from non-injecting routes of administration, NSW, Australia, 1992-1996. Addiction. 2000;95(4):569-73. 
55. Gossop M, Griffiths P, Powis B, Williamson S, Strang J. Frequency of non-fatal heroin overdose: survey of heroin users recruited in non-clinical settings. BMJ. $1996 ; 313(7054): 402$.

56. Smolka M, Schmidt LG. The influence of heroin dose and route of administration on the severity of the opiate withdrawal syndrome. Addiction. 1999;94(8):1191-8.

57. Gossop M, Griffiths $\mathrm{P}$, Strang J. Sex differences in patterns of drug taking behaviour. A study at a London community drug team. Br J Psychiatry. $1994 ; 164(1): 101-4$.

58. Anthony JC, Petronis KR. Early onset drug use and risk of later drug problems. Drug Alcohol Depend. 1995;40(1):9-15.

59. Cravioto P, Medina-Mora ME, de la Rosa B, Galvan F, Tapia-Conyer R. Patrones de consumo de heroína en una cárcel de la frontera norte de México: barreras de acceso a tratamiento. Salud Pública Méx. 2003;45(3):181-90.

60. De la Fuente L, Barrio G, Bravo MJ, Royuela L. Heroin smoking by "chasing the dragon": its evolution in Spain. Addiction. 1998; 93(3):444-6.

61. Gervin M, Hughes R, Bamford L, Smyth BP, Keenan E. Heroin smoking by "chasing the dragon" in young opiate users in Ireland: stability and associations with use to "come down" off "Ecstasy". J Subst Abuse

Treat. 2001;20(4):297-300.

62. Comer SD, Collins ED, MacArthur RB, Fischman MW. Comparison of intravenous and intranasal heroin self-administration by morphine-maintained humans.

Psychopharmacol (Berl). 1999;143(4):327-38.

63. De la Fuente L, Barrio G, Royuela L, Bravo MJ. The transition from injectin to smoking heroin in three Spanish cities. The Spanish group for the study of the route of heroin administration. Addiction. 1997;92:1749-63.

64. García Rodríguez O, Secades Villa R, Fernández Hermida JR. Comparación de pacientes cocainómanos y heroinómanos en el EuropASI. Adicciones.

2005;17(1):33-41.

65. Urrea A. Estudio descriptivo de pacientes que inician tratamiento en un P.M.M [tesis. Valencia: Universidad de Valencia; 1992.

66. Marina P. Adictos a opiáceos en Asturias: Un estudio de seguimiento [tesis]. Universidad de Cantabria, Santander: Facultad de Medicina; 1992.

67. Muga R, Sanvisens A, Bolao F, Tor J, Santesmases J, Pujol R, et al. Significant reductions of HIV prevalence but not of hepatitis $C$ virus infections in injection drug users from metropolitan Barcelona: 1987-2001. Drug Alcohol Depend. 2006;82 Suppl 1:S29-S33.

68. Bravo MJ, Barrio G, de la Fuente L, Colomo C, Royuela L, Grupo de Trabajo de Médicos del Mundo. Persistencia de conductas de riesgo para la transmisión del VIH en inyectores de drogas de Madrid, Sevilla y Valencia. Gac Sanit. 1999;13:109-18. 
69. Bravo MJ, Royuela L, Barrio G, Rodríguez-Arenas MA, de la Fuente L. Prevalencia de prácticas indirectas de compartir material para inyectarse drogas en Galicia, Madrid, Sevilla y Valencia. Gac Sanit. 2004;18:472-8.

Recibido: 16 de julio de 2009.

Aprobado: 7 de abril de 2010.

Guillermo Alonso Castaño Pérez. Fundación Universitaria Luis Amigó. Transversal 51 A N. ${ }^{\circ} 67$ B-90.Medellín, Colombia.

E-mail: farmacodependencia@funlam.edu.co 